For

\title{
Different Extraction Behaviors between Divalent and Trivalent Endohedral Metallofullerenes
}

Yongfu Lian, Zujin Shi, Xihuang Zhou and Zhennan Gu

Department of Chemistry, Peking University, Beijing 100871, P. R. China

Department of Chemistry, Heilongjiang University, Harbin 150080, P. R. China

guzn@chem.pku.edu.cn. 
Figure S1. The MALDI-TOF mass spectra of the raw soot containing (a) Sm, (b) Ba, (c) Ca, (d) Y, (e) La, and (f) Nd EMFs.
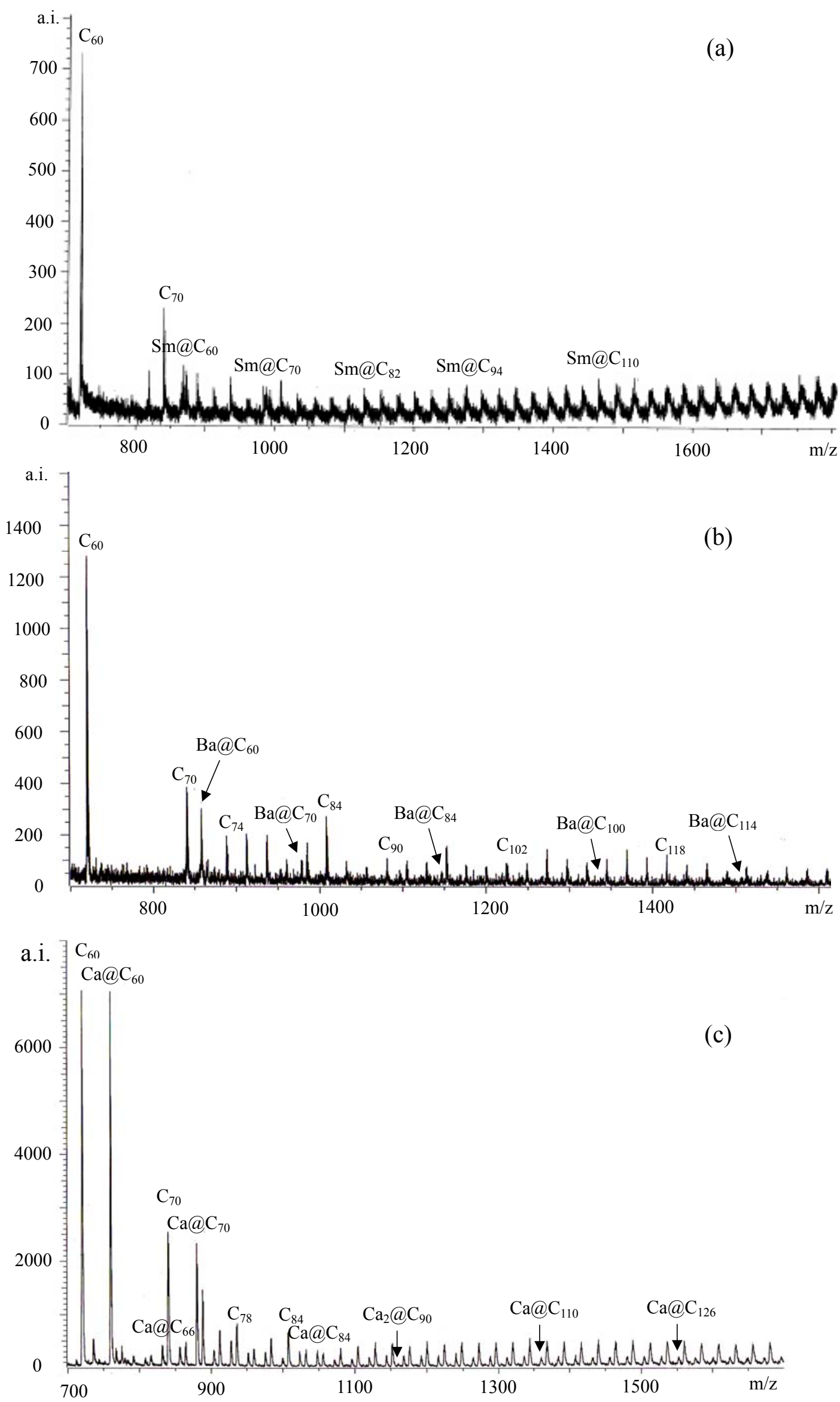

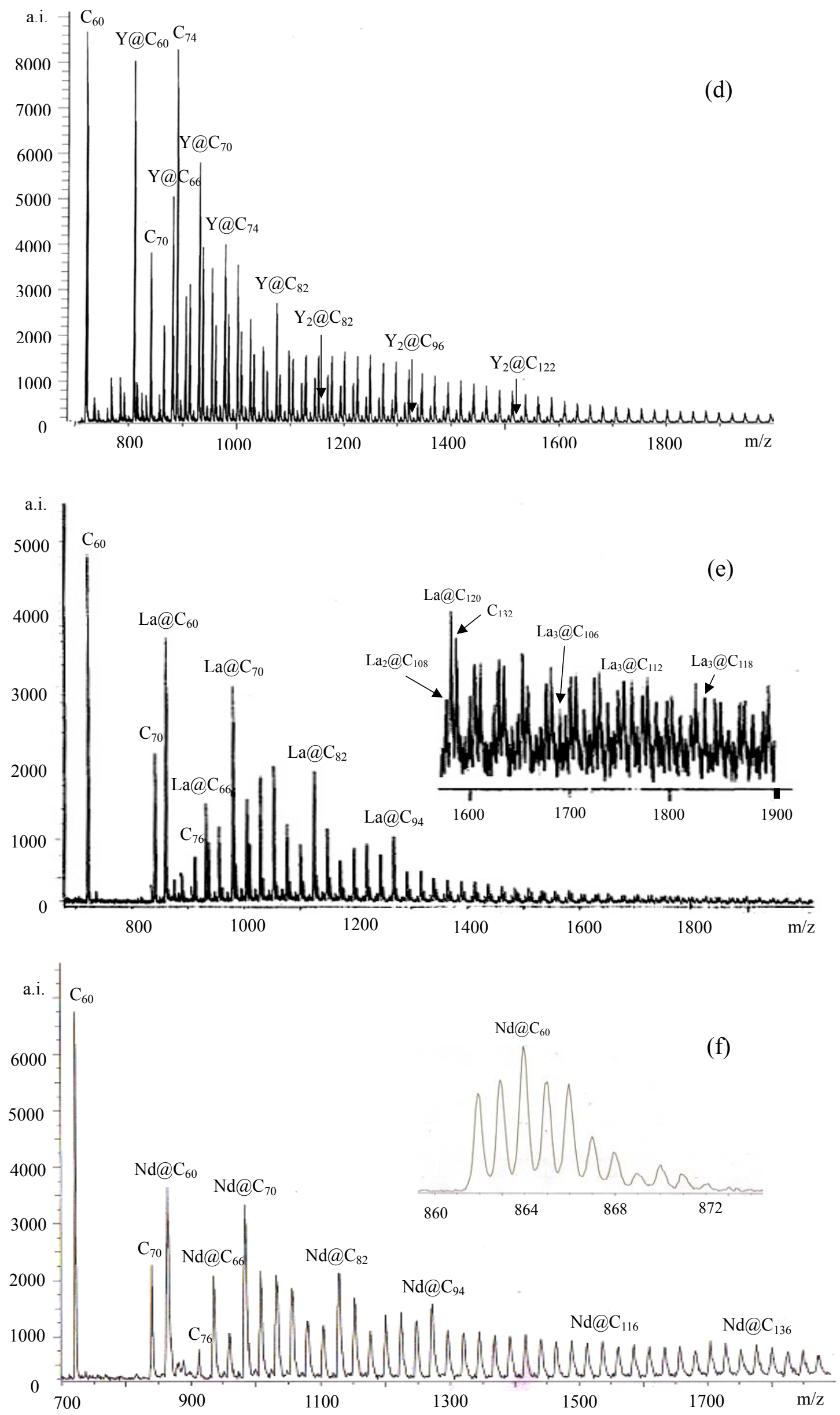
Figure S2. The MALDI-TOF mass spectra of the carbon disulfide extracts containing (a) Ba, (b) Sm, (c) Nd, (d) Y, and (e) La EMFs.
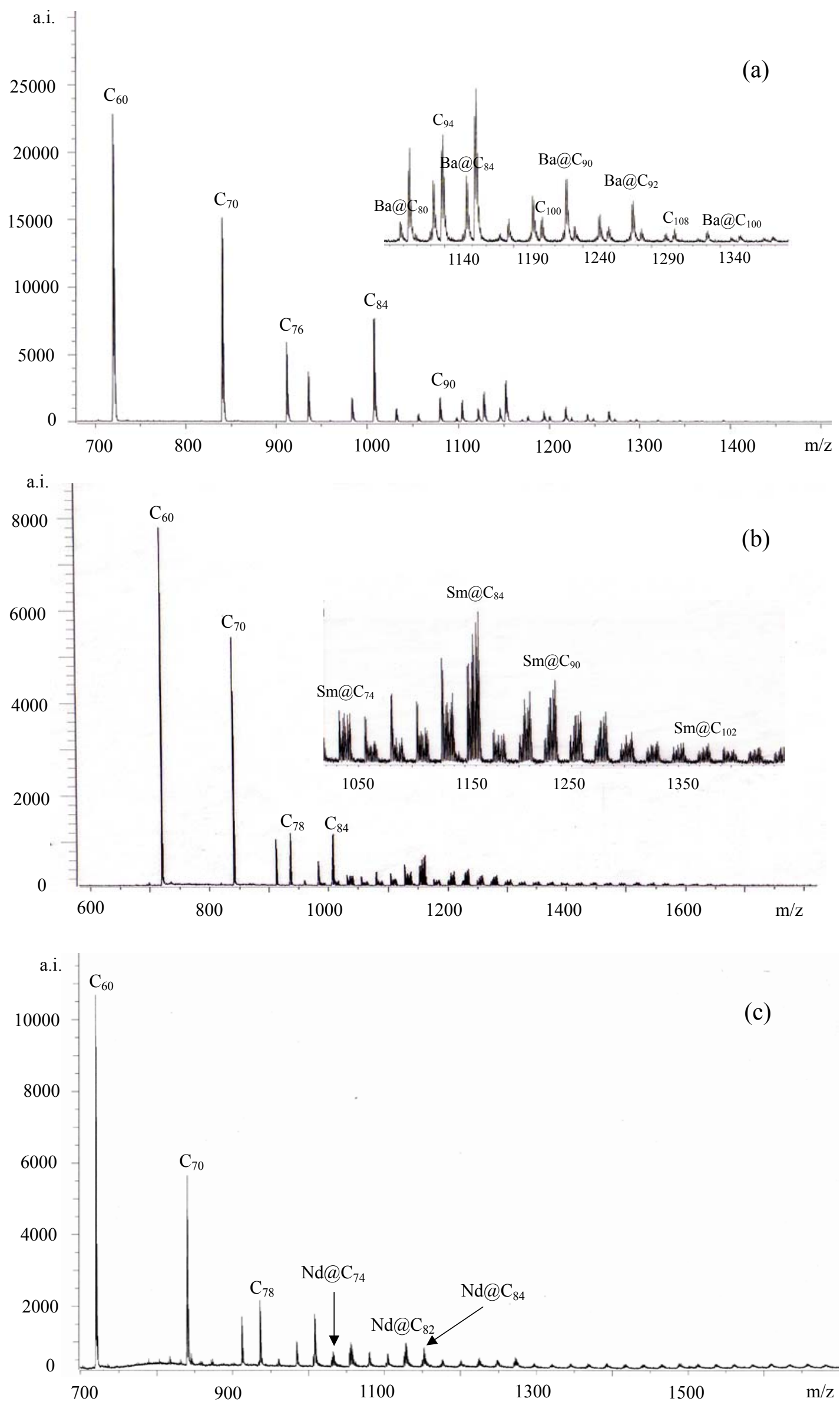

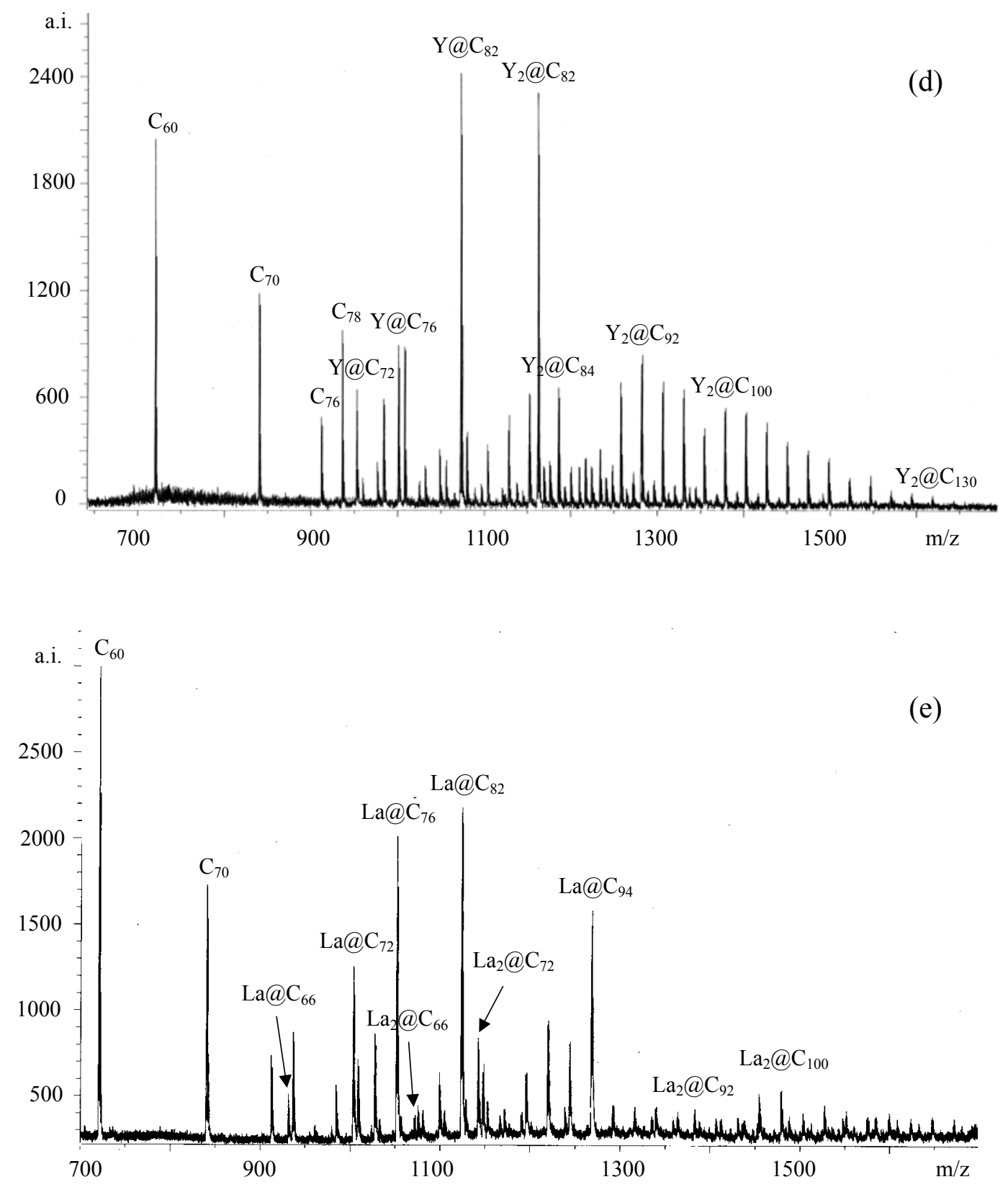
Figure S3. The MALDI-TOF mass spectra of the DMF extracts containing (a) Ba, (b) Ca, (c) Sm, (d) Nd, (e) Y, and (f) La EMFs.
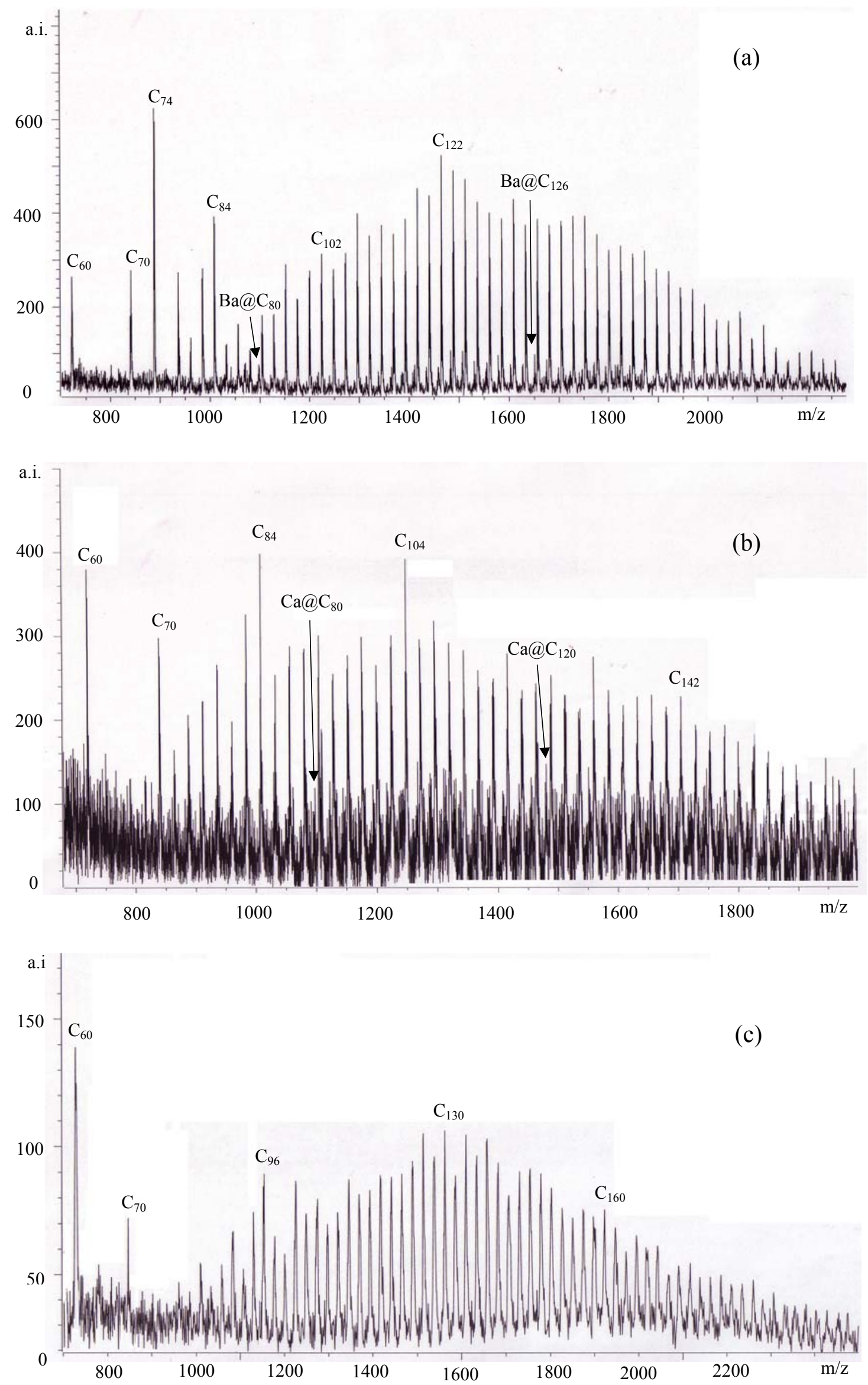

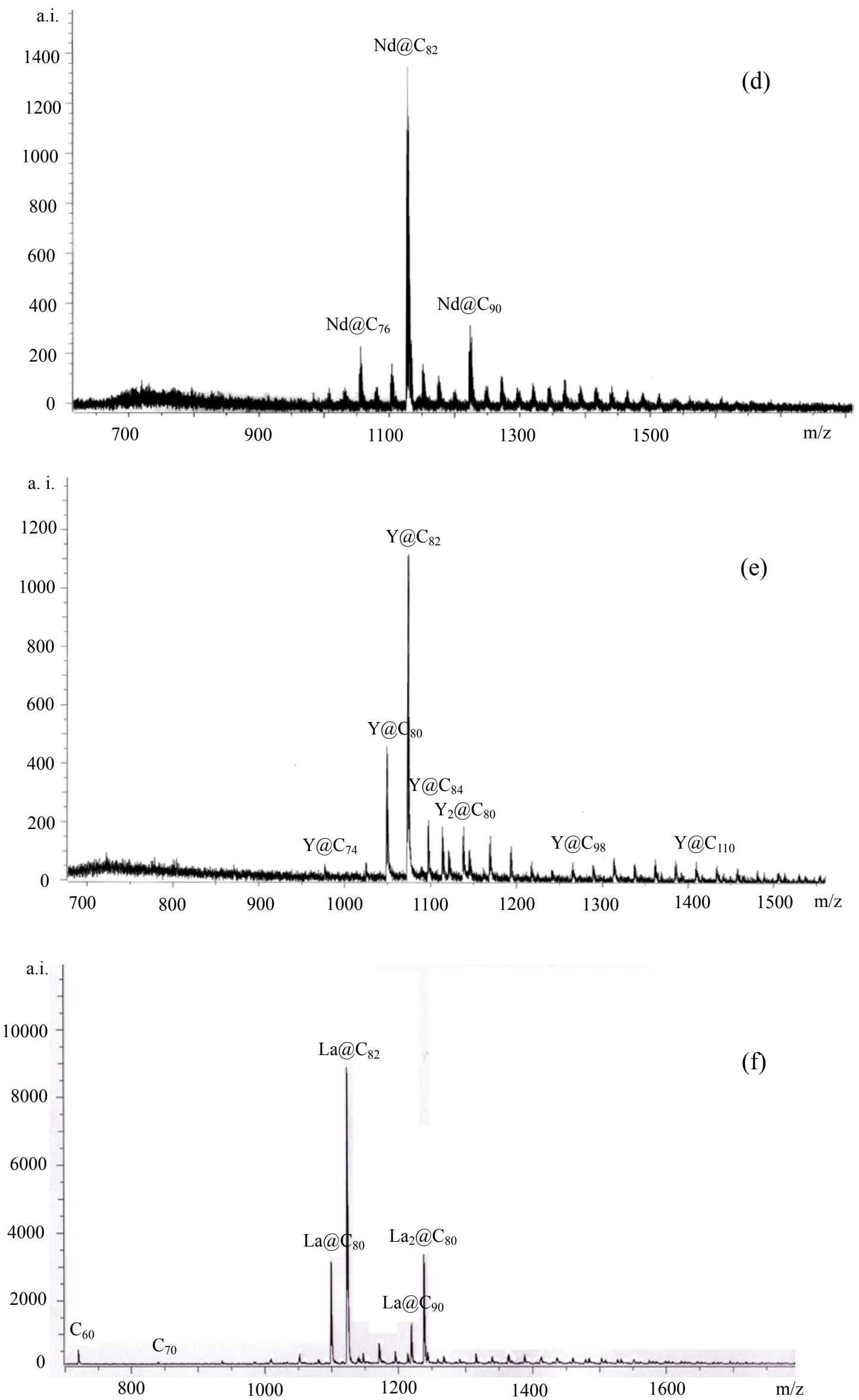
Figure S4. The MALDI-TOF mass spectra of the pyridine extracts containing (a) $\mathrm{Ba}$, (b) $\mathrm{Ca}$, (c) Sm, (d) Nd, (e) Y, and (f) La EMFs.
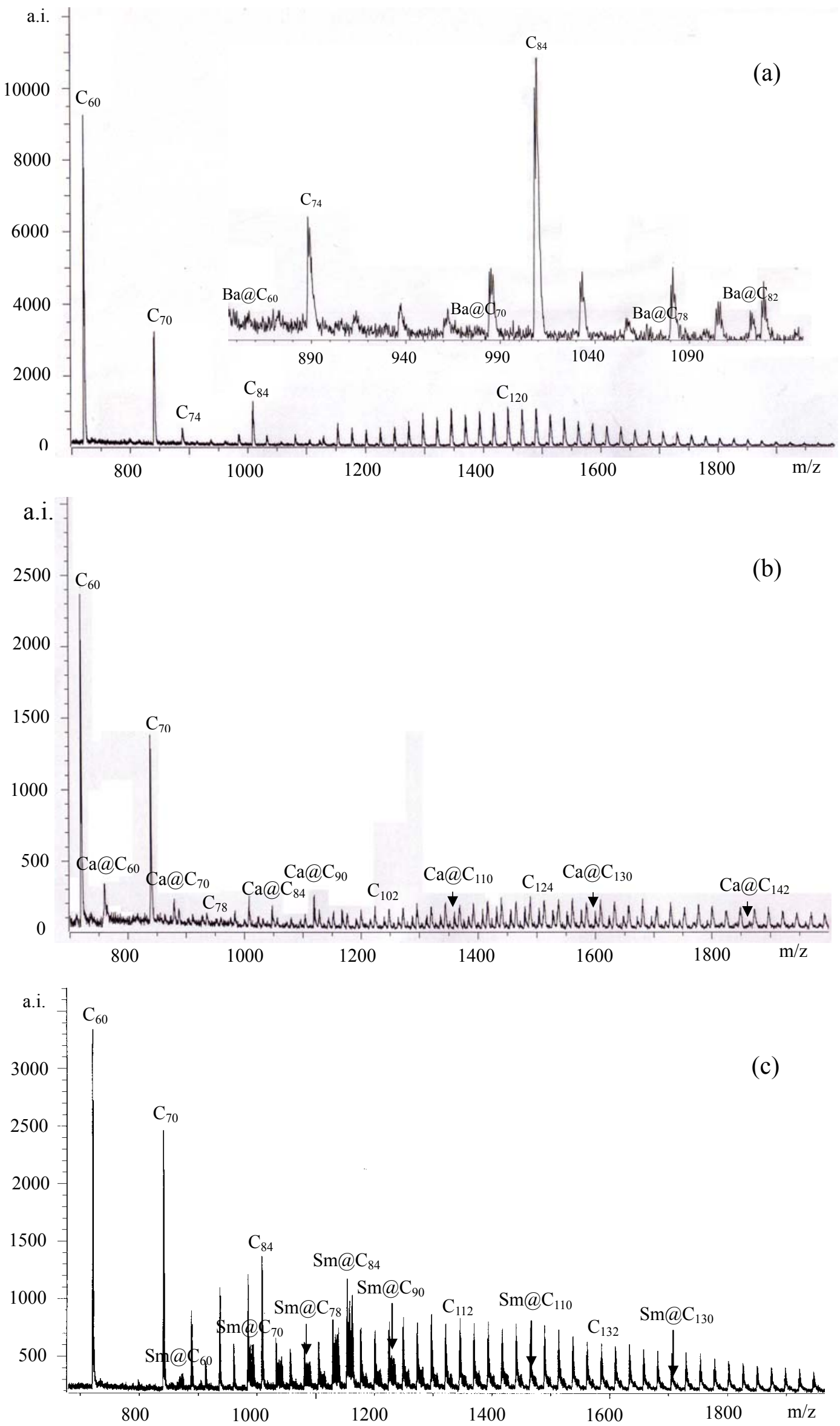

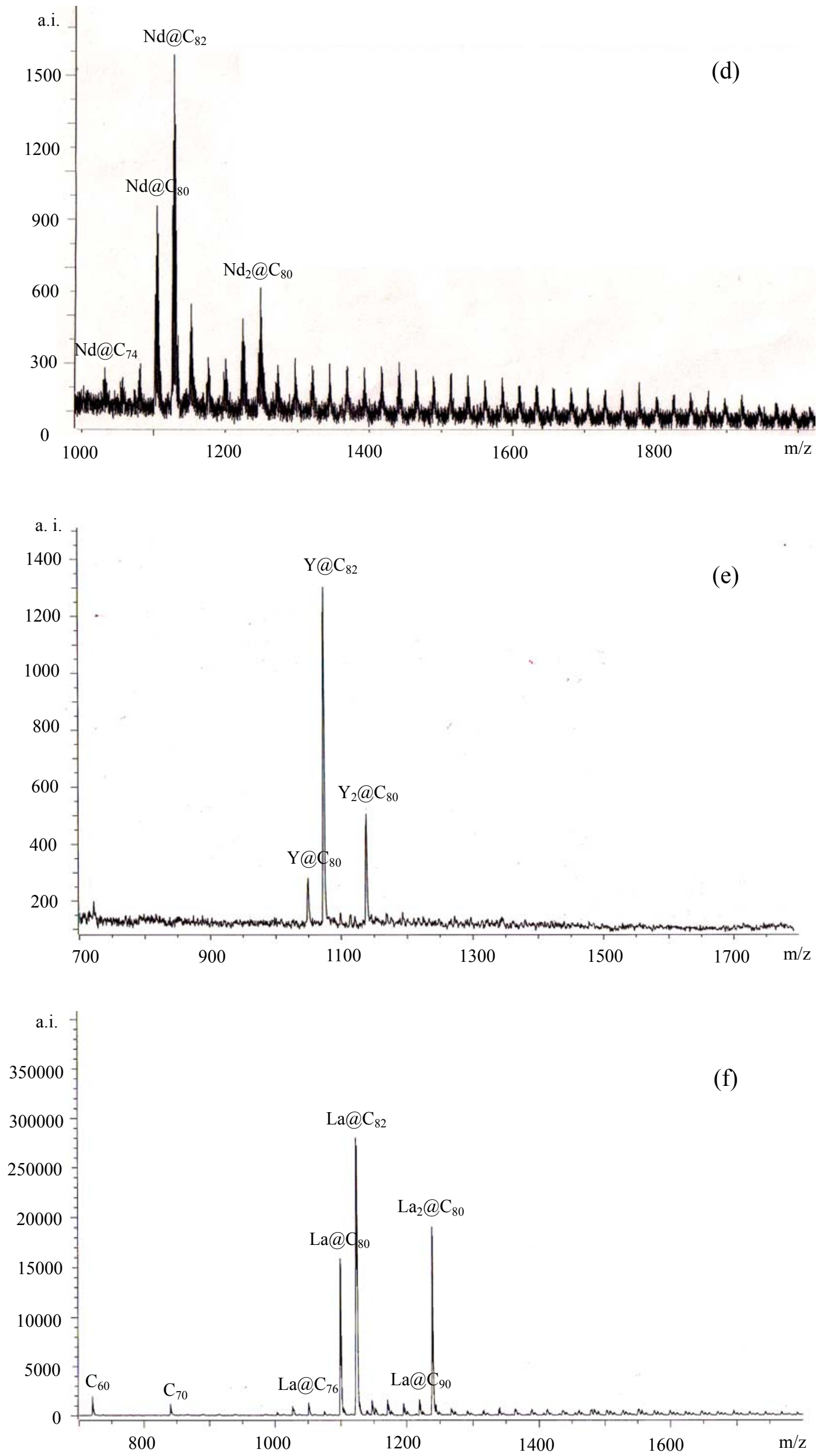
Figure S5. The MALDI-TOF mass spectra of the aniline extracts containing (a) $\mathrm{Ba}$, (b) $\mathrm{Ca}$, (c) Sm, (d) Gd, (e) Nd, and (f) La EMFs.
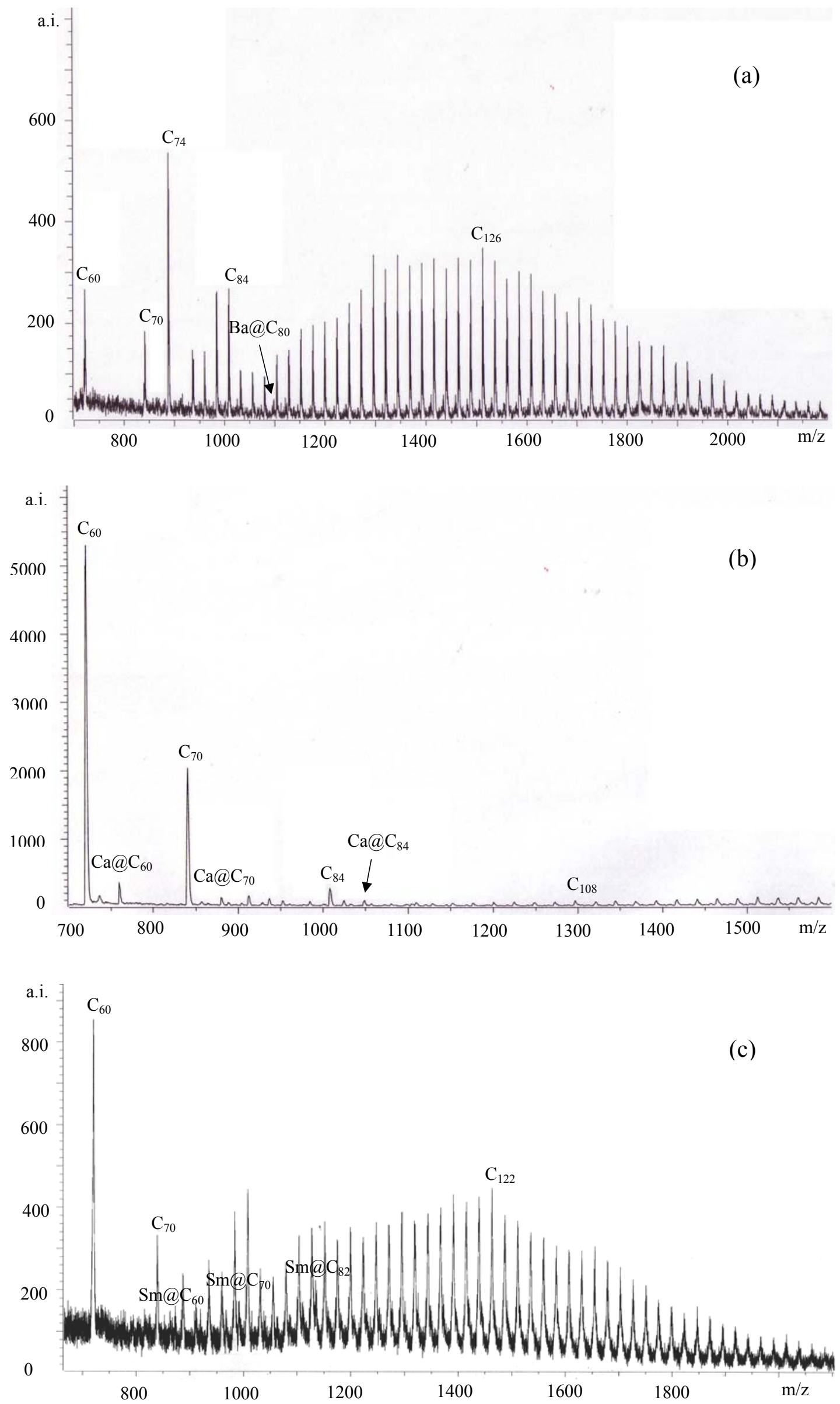

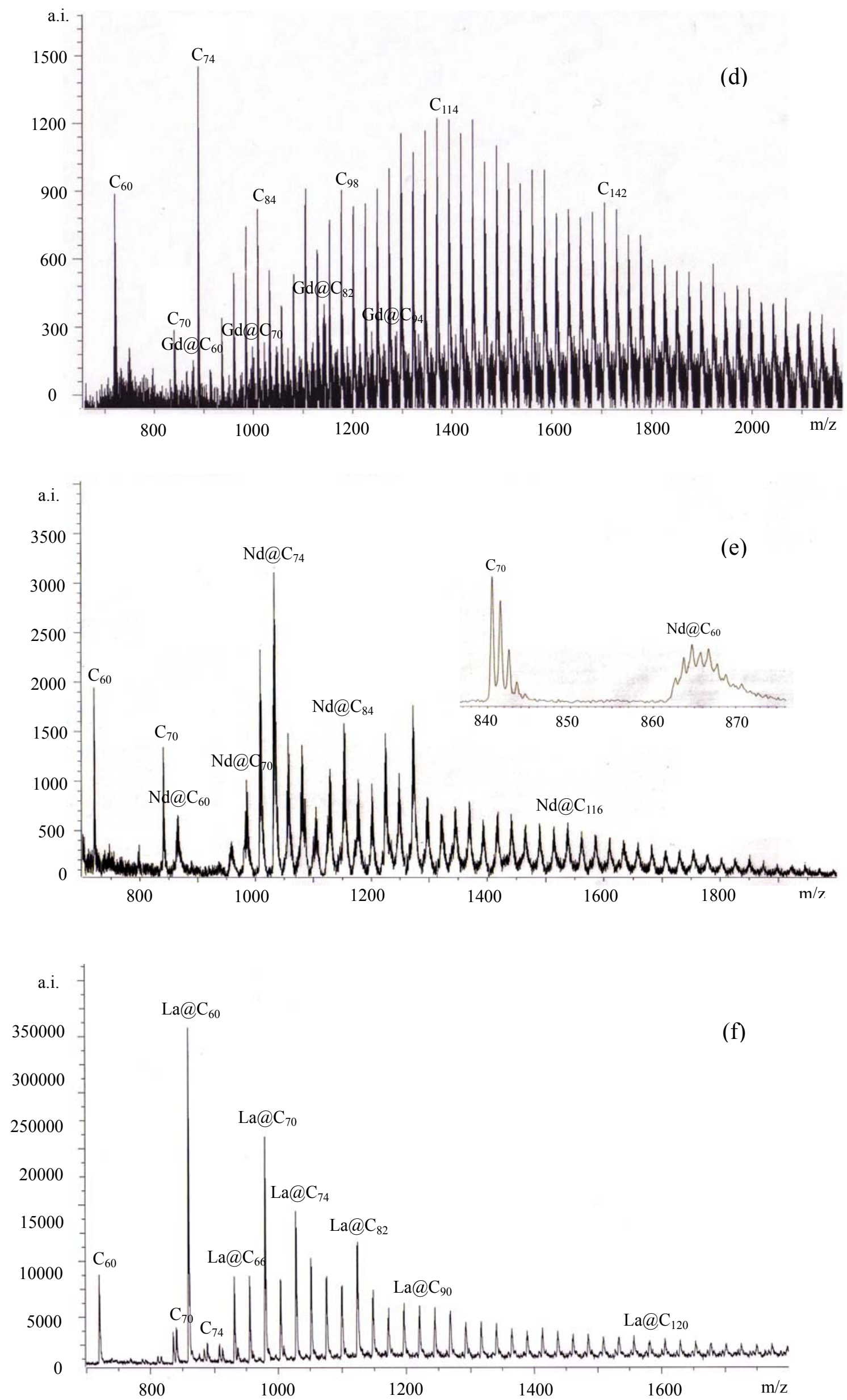